ARTICLE

Received 11 Apr 2014 | Accepted 18 Jun 2014 | Published 25 Jul $2014 \quad$ DOl: 10.1038/ncomms5446

\title{
Host iron status and iron supplementation mediate susceptibility to erythrocytic stage Plasmodium falciparum
}

Martha A. Clark¹, Morgan M. Goheen¹, Anthony Fulford ${ }^{2,3}$, Andrew M. Prentice ${ }^{2,3}$, Marwa A. Elnagheeb ${ }^{4}$, Jaymin Patel ${ }^{4}$, Nancy Fisher ${ }^{1}$, Steve M. Taylor ${ }^{5}$, Raj S. Kasthuri ${ }^{6} \&$ Carla Cerami $^{4}$

Iron deficiency and malaria have similar global distributions, and frequently co-exist in pregnant women and young children. Where both conditions are prevalent, iron supplementation is complicated by observations that iron deficiency anaemia protects against falciparum malaria, and that iron supplements increase susceptibility to clinically significant malaria, but the mechanisms remain obscure. Here, using an in vitro parasite culture system with erythrocytes from iron-deficient and replete human donors, we demonstrate that Plasmodium falciparum infects iron-deficient erythrocytes less efficiently. In addition, owing to merozoite preference for young erythrocytes, iron supplementation of iron-deficient individuals reverses the protective effects of iron deficiency. Our results provide experimental validation of field observations reporting protective effects of iron deficiency and harmful effects of iron administration on human malaria susceptibility. Because recovery from anaemia requires transient reticulocytosis, our findings imply that in malarious regions iron supplementation should be accompanied by effective measures to prevent falciparum malaria.

\footnotetext{
${ }^{1}$ Department of Microbiology and Immunology, University of North Carolina, Chapel Hill, North Carolina 27599, USA. ${ }^{2}$ Medical Research Council International Nutrition Group, London School of Hygiene and Tropical Medicine, London WC1E 7HT, UK. ${ }^{3}$ Medical Research Council, Keneba, The Gambia. ${ }^{4}$ Department of Epidemiology, Gillings School of Global Public Health, University of North Carolina, Chapel Hill, North Carolina 27599 , USA. ${ }^{5}$ Division of Infectious Diseases and International Health, Duke University Medical Center, Durham, North Carolina 27710, USA. 6 Division of Hematology Oncology, Department of Medicine, University of North Carolina, Chapel Hill, North Carolina 27599, USA. Correspondence and requests for materials should be addressed to C.C. (e-mail: ccerami@unc.edu).
} 
T he interactions between falciparum malaria and iron deficiency anaemia (IDA) are complex and bi-directional. Malaria causes acute anaemia by destroying both infected and uninfected red blood cells $(\mathrm{RBCs})^{1}$, whereas persistent sub-clinical infection causes a milder anaemia of infection by blocking iron recycling to the bone marrow ${ }^{2}$. Conversely, once established, IDA protects both pregnant women ${ }^{3-5}$ and children $^{6-8}$ from malaria. In addition, supplemental iron, given alone or in combination with other micronutrients, predisposes children to malaria ${ }^{8,9}$ and other serious adverse outcomes ${ }^{10}$. Iron homeostasis has been implicated in regulating liver stage $P$. falciparum infection; in murine studies, erythrocytic stage malaria infection initiates hepcidin-mediated hepatic hypoferremia, which blocks superinfections by sporozoites from competing plasmodial strains ${ }^{11}$. Mathematical modelling suggests that this can explain the low levels of superinfections in young children ${ }^{11}$, but this mechanism cannot account for observed reductions in the risk of primary malaria infection in children with IDA. It has also been speculated that transient peaks in nontransferrin-bound iron caused by administration of highly absorbable iron supplements ${ }^{12}$ could promote intra-erythrocytic parasite growth ${ }^{13}$ or bacterial septicemia (a common cause of death in malaria patients ${ }^{14-16}$ ) but definitive evidence is absent.

As iron deficiency and iron supplementation of iron deficient individuals profoundly alters erythropoiesis, RBC physiology, and RBC population structure, we hypothesized that iron deficiency and iron supplementation directly impact the disease causing erythrocytic stage of $P$. falciparum infection. In our investigations, we minimize the confounding factors that have complicated prior field studies of the relationship between host iron status, iron supplementation and falciparum malaria by utilizing an in vitro system with freshly isolated RBCs from donors with well-defined, physiologic iron states recruited through our US-based hospital clinic. This approach eliminated the influence of acquired and innate immunity to malaria, haemoglobinopathies and concurrent inflammation. Our study reveals that RBCs from donors with IDA confer malaria protection by impairing P. falciparum invasion and intra-erythrocyte propagation. This protective effect was reversed when donors with IDA received iron supplementation. We go on to show that when iron-deficient RBCs are replaced with iron-replete (IR) RBCs in vitro (as occurs in individuals with IDA following iron supplementation) the susceptibility to $P$. falciparum infection is increased. These findings support well-described clinical patterns of differential susceptibility to malaria. Taken together, they indicate that therapeutic iron supplementation conspires with host iron status to mediate host RBC susceptibility to malaria infection by altering the dynamic structure of the host's RBC population.

\section{Results}

Malaria growth is reduced in RBCs from individuals with IDA. To determine the effect of IDA on the growth of erythrocytic stage $P$. falciparum, we enrolled donors with and without IDA from a non-malaria endemic area through our US-based hospital clinic. Donors were classified as IR (haemoglobin (Hgb) $>11 \mathrm{~g} \mathrm{dl}^{-1}$, mean corpuscular volume $(\mathrm{MCV})>80 \mathrm{fL}$, ferritin $>12 \mathrm{ng} \mathrm{ml}^{-1}$ ) or as IDA $\left(\mathrm{Hgb}<11 \mathrm{~g} \mathrm{dl}^{-1}, \mathrm{MCV}<80 \mathrm{fL}\right.$, ferritin $<12 \mathrm{ng} \mathrm{ml}^{-1}$ ) (Table 1). Non-anaemic donors with lowiron stores $\left(\mathrm{Hgb}>11 \mathrm{~g} \mathrm{dl}^{-1}\right.$, ferritin $<12 \mathrm{ng} \mathrm{ml}^{-1}$ ) were excluded. P. falciparum (strains 3D7, Dd2 and FCR3-FMG) were grown in either RBCs from the IR $(n=10)$ or IDA $(n=7)$ donors in up to three consecutive $96 \mathrm{~h}$ growth assays (Supplementary Fig. 1). We observed that parasite growth rates were reduced in RBCs from IDA donors as compared with growth in RBCs from IR donors by $48.8 \%$ (standard deviation (s.d.) \pm 23.9 ), $34.3 \%$ (s.d. \pm 22.2 ) and $50.0 \%$ (s.d. \pm 20.4 ) for strains $3 \mathrm{D} 7$, Dd2 and FCR3-FMG, respectively (Fig. 1a). These findings clearly show that $P$. falciparum propagation is reduced within RBCs from IDA individuals, but that variability may exist in the degree to which different $P$. falciparum isolates are affected by IDA.

Malaria growth is increased in RBCs from iron-supplemented donors. Given field evidence that supplementation of children with $12.5 \mathrm{mg}$ of iron $\left(1-1.5 \mathrm{mg} \mathrm{kg}^{-1}\right)$ and $50 \mathrm{ng}$ of folic acid may potentiate the risk of malaria ${ }^{9}$, we next investigated the effects of

Table 1 | Iron parameters and values of study participants.

\begin{tabular}{|c|c|c|c|c|c|c|}
\hline Variable & $\begin{array}{l}\text { Normal } \\
\text { range }\end{array}$ & IDA $(N=7)$ & $\begin{array}{l}\text { IDA }+ \text { Fe } \\
(N=6)\end{array}$ & $\operatorname{IR}(N=10)$ & $\begin{array}{c}\text { IR after } 1 \text { month } \\
\text { iron supplementation } \\
(\text { IR }+\mathrm{Fe} ; \boldsymbol{N}=\mathbf{4})\end{array}$ & $\begin{array}{l}\text { IR after } 2 \text { months } \\
\text { iron supplementation } \\
\quad(I R+F e ; \mathbf{N}=\mathbf{4})\end{array}$ \\
\hline White blood cell ( $\times 10^{9}$ per I) & $4.5-11.0$ & $4.84(1.78)$ & $4.45(1.15)$ & $6.40(1.61)$ & $6.30(1.41)$ & $6.20(1.12)$ \\
\hline Red blood cell $\left(\times 10^{12}\right.$ per I) & $4.0-5.2$ & $3.96(0.60)$ & $3.42(0.35)$ & $4.78(0.52)$ & $4.94(0.52)$ & $4.91(0.39)$ \\
\hline Haemoglobin $\left(\mathrm{g} \mathrm{dl}^{-1}\right)$ & $12.0-16.0$ & $8.20(1.56)$ & $8.73(1.23)$ & $14.60(1.40)$ & $14.90(0.57)$ & $14.63(0.53)$ \\
\hline Haematocrit (\%) & $36.0-46.0$ & $28.13(4.32)$ & $29.11(4.25)$ & $42.80(4.31)$ & $43.85(2.62)$ & $42.88(2.81)$ \\
\hline Mean corpuscular volume $(\mathrm{fL})$ & $80.0-100.0$ & $71.20(6.63)$ & $84.78(8.28)$ & $89.80(2.74)$ & $89.67(4.24)$ & $87.75(2.50)$ \\
\hline $\begin{array}{l}\text { Mean corpuscular haemoglobin } \\
\text { (Pg) }\end{array}$ & $26.0-34.0$ & $20.79(2.54)$ & $25.50(2.35)$ & $30.70(0.95)$ & $30.50(2.12)$ & $30.0(1.83)$ \\
\hline $\begin{array}{l}\text { Mean corpuscular haemoglobin } \\
\text { concentration }\left(\mathrm{g} \mathrm{dl}{ }^{-1}\right)\end{array}$ & $31.0-37.0$ & $29.20(1.50)$ & $30.27(0.86)$ & $34.20(0.63)$ & $34.50(0.71)$ & $34.25(0.96)$ \\
\hline Red cell distribution width (\%) & $12.0-15.0$ & $17.41(1.44)$ & $18.13(2.71)$ & $13.22(0.85)$ & $13.90(0.28)$ & $13.20(0.45)$ \\
\hline Mean platelet volume (fL) & 7.0-10.0 & $8.56(1.05)$ & $8.78(0.65)$ & $7.63(0.54)$ & $8.45(0.92)$ & $8.15(0.52)$ \\
\hline Platelet count $\left(\times 10^{9}\right.$ per I) & $150.0-440.0$ & $294.57(59.70)$ & $344.0(168.63)$ & $251.80(36.70)$ & $212.50(89.80)$ & $256.0(79.31)$ \\
\hline Iron total $\left(\mathrm{mg} \mathrm{dl}^{-1}\right)$ & $35.0-165.0$ & $21.60(10.55)$ & $32.0(16.79)$ & $103.60(43.34)$ & $105.75(32.71)$ & $87.50(17.99)$ \\
\hline Transferrin $\left(\mathrm{mg} \mathrm{dl}^{-1}\right)$ & $200.0-380.0$ & $343.60(74.72)$ & $305.20(63.55)$ & $264.30(41.33)$ & $275.0(29.50)$ & $276.75(31.83)$ \\
\hline $\begin{array}{l}\text { Transferrin iron binding } \\
\text { capacity }\left(\mathrm{mg} \mathrm{dl}^{-1}\right)\end{array}$ & $252.0-479.0$ & $438.67(92.08)$ & $384.60(80.29)$ & $333.0(52.20)$ & $346.0(37.43)$ & $348.75(40.07)$ \\
\hline Transferrin saturation (\%) & $15.0-50.0$ & $4.71(3.15)$ & $8.0(7.45)$ & $31.60(13.04)$ & $31.0(9.49)$ & $25.75(7.63)$ \\
\hline Ferritin $(\mathrm{ng} \mathrm{ml}-1)$ & $30.0-151.0$ & $5.71(2.75)$ & $17.17(25.44)$ & $42.01(24.28)$ & $46.75(38.75)$ & $33.33(18.45)$ \\
\hline Reticulocyte (\%) & $0.5-2.7$ & $1.42(0.55)$ & $3.52(1.62)$ & $1.48(0.48)$ & $2.40(0.71)$ & $1.40(0.38)$ \\
\hline
\end{tabular}


iron supplementation of IDA and IR individuals on in vitro growth of erythrocytic stage $P$. falciparum. We first collected RBCs from IDA patients who were receiving iron supplementation (IDA $+\mathrm{Fe})$; these individuals met the above criteria for IDA and were receiving either high-dose oral ferrous sulfate $(60 \mathrm{mg}$ elemental iron orally three times per day (9$\left.12.6 \mathrm{mg} \mathrm{kg}^{-1}\right)$ ) or intravenous iron (at a dosage determined by their personal physician using the following equation: Dose $=0.0442$ [desired Hgb - observed $\mathrm{Hgb}] \times \mathrm{LBW}+[0.26 \times$ $\mathrm{LBW}]$ ). IDA $+\mathrm{Fe}$ group $\mathrm{Hgb}$ values ranged from 6.6 to $9.8 \mathrm{~g} \mathrm{dL}^{-1}$ and MCV values ranged from 75 to $98 \mathrm{fL}$. Additionally reticulocyte counts and red cell distribution width (RDW) were elevated; and average mean corpuscular haemaglobin concentration (MCHC), total iron, and ferritin values were greater than that of the IDA group but still lower than that of the IR group (Table 1). Together, these values are indicative of an erythropoietic response to iron supplements, but not full recovery from IDA. Comparison of the growth rate of $P$. falciparum (strains 3D7, Dd2 and FCR3-FMG) within RBCs from the IDA + Fe donors to the growth rate of parasites within RBCs from IR donors revealed increases in P. falciparum growth of $17.3 \%$ (s.d. \pm 22.7 ), $17.6 \%$ (s.d. \pm 14.0 ) and $26.3 \%$ (s.d. \pm 16.1 ) for 3D7, Dd2 and FCR3-FMG in RBCs from IDA + Fe donors (Fig. 1b).
We additionally assessed the effect of iron supplementation of IR individuals on $P$. falciparum growth. For this study, IR individuals donated blood at enrollment (baseline) and were then prescribed daily oral iron supplementation $(325 \mathrm{mg}$ ferrous sulfate). Iron-supplemented IR study participants (IR $+\mathrm{Fe}$ ) subsequently returned at one and two months following initiation of daily iron supplementation to donate blood. At each donation (enrollment, 1 month and 2 months), the growth rate of P. falciparum (strains 3D7, Dd2 and FCR3-FMG) within RBCs from IR + Fe donors was determined and then compared with the corresponding parasite growth rates within RBCs from a nonsupplemented IR donor. Compared with RBCs from IR donors, we observed in RBCs from $\mathrm{IR}+\mathrm{Fe}$ donors that 1 month of iron supplementation increases of $17.5 \%$ (s.d. \pm 16.1 ), $11.3 \%$ (s.d. \pm 15.7 ) and $6.6 \%$ (s.d. \pm 8.1 ) in growth for $3 \mathrm{D} 7, \mathrm{Dd} 2$ and FCR3-FMG, respectively. There was no change in parasite growth rate in RBCs collected 1 and 2 months after administering iron supplements (Fig. 1c). Analyses of Hgb, haematocrit, MCV, MCHC, transferrin saturation, ferritin and reticulocyte count of $\mathrm{IR}+\mathrm{Fe}$ donors revealed no significant change in their iron status following iron supplementation (Table 1).

To comprehensively compare and ultimately quantify the impact of IDA and iron supplementation on the growth of $P$. falciparum in vitro, we integrated data from all growth
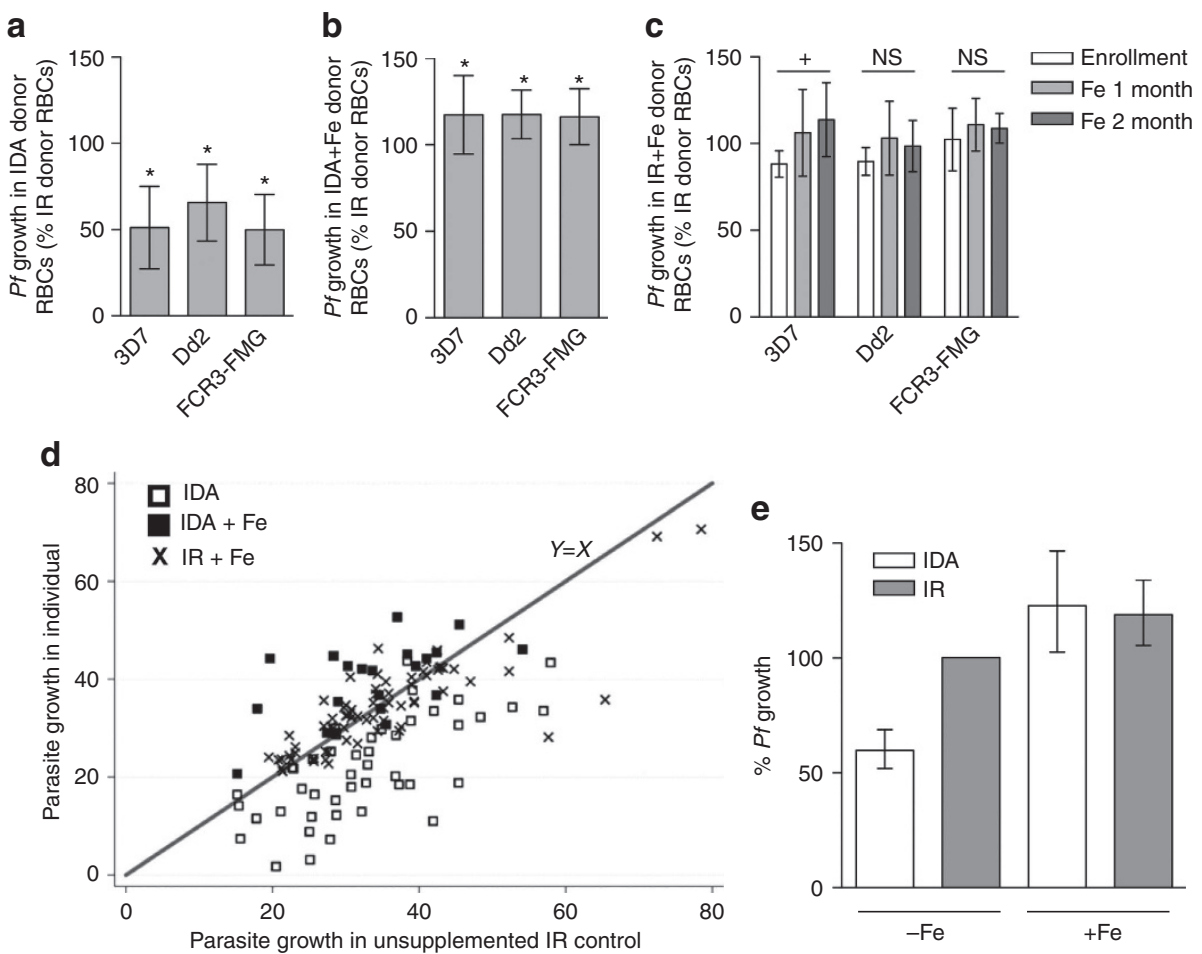

Figure 1 | P. falciparum growth is reduced in iron-deficient RBCs and iron supplementation eliminates growth attenuation. (a-c) Growth experiments with RBCs from IDA donors $(n=7)$, IDA + Fe donors $(n=5)$ and IR + Fe donors $(n=4)$ were performed. Growth rate in RBCs from an IR donor served as the control. Bars represent growth of $P$. falciparum (strains 3D7, Dd2 and FCR3-FMG) in indicated RBCs, normalized to growth in RBCs from IR donors (\% Pf growth in IR RBC). Error bars represent the s.d. (a) Bars represent growth in RBCs from IDA donors. Significance was determined by two-tailed paired Student's $t$-test. ${ }^{\star} P<3 \mathrm{E}^{-10}$ as compared with $P$. falciparum growth in RBCs from IR donors. (b) Bars represent growth in RBCs from IDA + Fe donors. Significance was determined by two-tailed paired Student's $t$-test. ${ }^{\star} P<0.0003$ as compared with $P$. falciparum growth in RBCs from IR donors. (c) Bars represent growth of $P$. falciparum in RBCs from IR + Fe donors at enrollment, 1 month and 2 months on iron, normalized to growth in RBCs from IR donors (\% Pf growth in IR RBC). Significance was determined by one-way analysis of variance. ${ }^{\dagger} P<0.02$ for strain $3 D 7$ and nonsignificant (n.s.) for strains Dd2 and FCR3-FMG. (d) Mean growth rate of $P$. falciparum in RBCs from each individual IDA ( $\square$ ), IR + Fe (x) and IDA + Fe ( $\mathbf{\square})$ donor plotted against the growth rate of $P$. falciparum in corresponding control RBCs from non-supplemented IR donors. Data were analysed by mixed effects regression. The $Y=X$ line was fit to the growth rates (in RBCs from IR donors). Points below the $Y=X$ line indicate growth rates less than that within RBCs from IR donors. (e) Graphical summary of the mixed effects regression analysis shown in $\mathbf{d}$. Donor and parasite preparation were fitted as crossed random effects. The bars show the estimated parasite growth of $P$. falciparum in RBCs from the IDA, IDA + Fe and IR + Fe donors as a percent of $P$. falciparum growth in RBCs from nonsupplemented IR donors. Error bars represent the $95 \%$ confidence interval. 


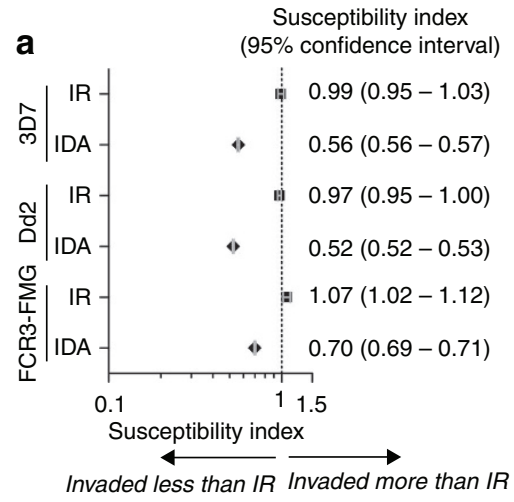

C

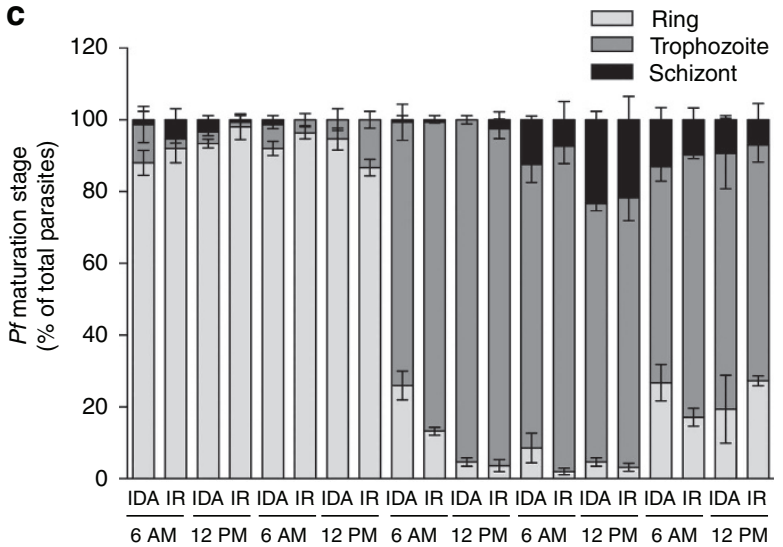

b

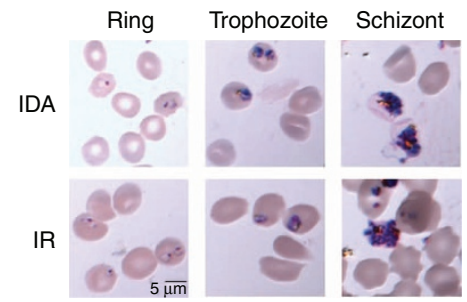

d

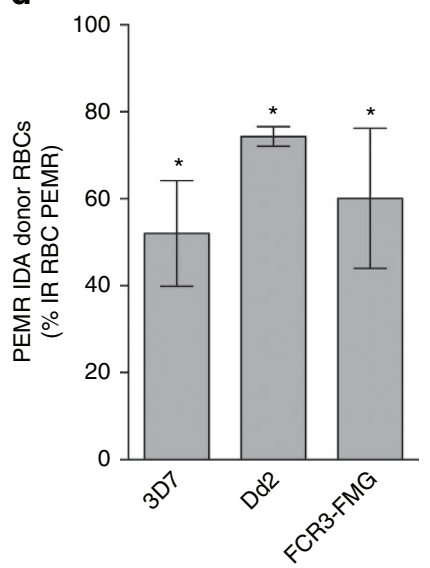

Figure 2 | P. falciparum invasion and growth are reduced in RBCs from IDA donors. (a) Direct comparison of invasion into RBCs from either IDA or IR donors. Invasion experiments for RBCs from all IDA donors were performed independently and each experiment was performed in triplicate. Data show the mean SI of six independent experiments performed with RBCs from six IDA donors. The marker represents the SI point estimate and the bar represents the $95 \% \mathrm{Cl}$. An SI of 1.0 indicates no difference in parasite invasion of the two RBC populations. (b,c) Comparison of the maturation of $P$. falciparum in RBCs donated by IDA and IR donors. Geimsa-stained thin blood smears were made every $6 \mathrm{~h}$ and 1,000 RBCs were counted by light microscopy to determine the percent of pRBCs as well as parasite intra-erythrocytic stage of maturation. Data are from a representative experiment (with strain FCR3-FMG) of three independent experiments performed with RBCs from three IDA donors infected with either P. falciparum strain 3D7, Dd2or FCR3. (b) Giemsa-stained thin blood smears of $P$. falciparum ring, trophozoite and schizont stage parasites in RBCs from an IDA and an IR donor. (c) Bars indicate percent frequency of parasite ring, trophozoite and schizont stages in RBCs from an IDA and IR donor at each $6 \mathrm{~h}$ time point. Error bars represent the s.d. (d) Comparison of the parasite erythrocyte multiplication rate (PEMR) of $P$. falciparum within RBCs from IDA and IR donors. Bars represent PEMR of $P$. falciparum in RBCs from IDA donors, normalized to the PEMR of parasites in RBCs from IR donors (\% IR PEMR). Data are the mean of three independent experiments performed in triplicate with RBCs from three IDA donors. Error bars represent the s.d. Significance determined by two-tailed paired Student's $t$-test. ${ }^{\star} P<3 E^{-6}$, compared with PEMR in RBCs from IR donors.

experiments and fit a multilevel random effects model to the pooled data (Fig. 1d). The outputs of this analysis were growth rates of any parasite strain in experimental RBCs plotted against the growth rate in IR RBC controls. Values were adjusted for variation between study participants, day-to-day differences in parasite preparations and differences in $P$. falciparum strain growth rates. The $\mathrm{Y}=\mathrm{X}$ line was fit to the growth rate of $P$. falciparum in RBCs from IR donors. Data above the $\mathrm{Y}=\mathrm{X}$ line indicate growth rates greater than that of parasite growth in RBCs from IR donors and data below the $\mathrm{Y}=\mathrm{X}$ line indicate growth rates lower than that of parasite growth RBCs from IR donors. Based on this analysis, we estimate that compared with IR RBCs, P. falciparum growth is reduced (59.8\% (95\% confidence interval $(\mathrm{CI})=51.9-68.8))$ in RBCs from IDA donors, and that there is a slight increase in the growth of $P$. falciparum in RBCs from IDA + Fe donors $(22.8 \%(95 \% \mathrm{CI}=2.7-46.7))$ and $\mathrm{RBCs}$ from $\mathrm{IR}+\mathrm{Fe}$ donors $(18.9 \%(95 \% \mathrm{CI}=5.0-33.9))$; no difference was observed between $P$. falciparum growth in RBCs from IR $+\mathrm{Fe}$ donors and IDA + Fe donors (Fig. 1e). These data clearly indicate that IDA substantially attenuates the growth of $P$. falciparum parasites and that iron supplementation of donors with IDA reverses the protection provided by IDA against falciparum infection. Furthermore, these data suggest that iron supplementation of IR individuals may slightly increase propagation of erythrocytic stage P. falciparum.

RBCs from donors with IDA are refractory to malaria infection. Propagation of the erythrocytic stage of P. falciparum may be impeded at the point of (i) invasion, (ii) maturation or (iii) production of infectious daughter merozoites. To determine why P. falciparum infection of RBCs from IDA donors is reduced, we systematically assessed the capacity of $P$. falciparum to progress through each of these rate-limiting steps within $\mathrm{RBCs}$ from IDA donors compared with RBCs from IR donors. To assess invasion, we directly compare invasion of $P$. falciparum strains 3D7, Dd2 and FCR3-FMG into RBCs from IDA and IR donors with a barcoded RBC flow cytometry based invasion assay. To express the differential invasion of RBCs, we computed the susceptibility index (SI), which is the ratio of the relative risk of 
a

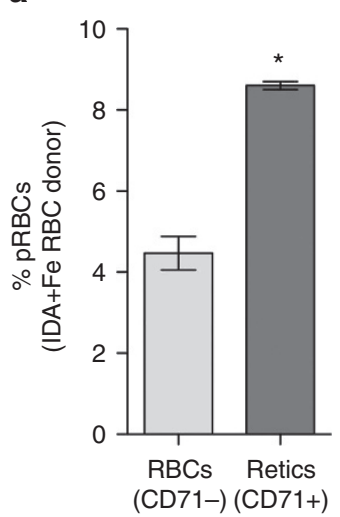

b

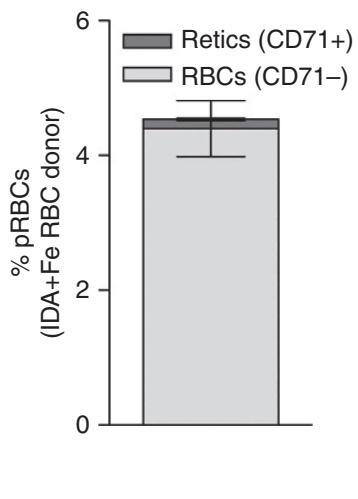

C

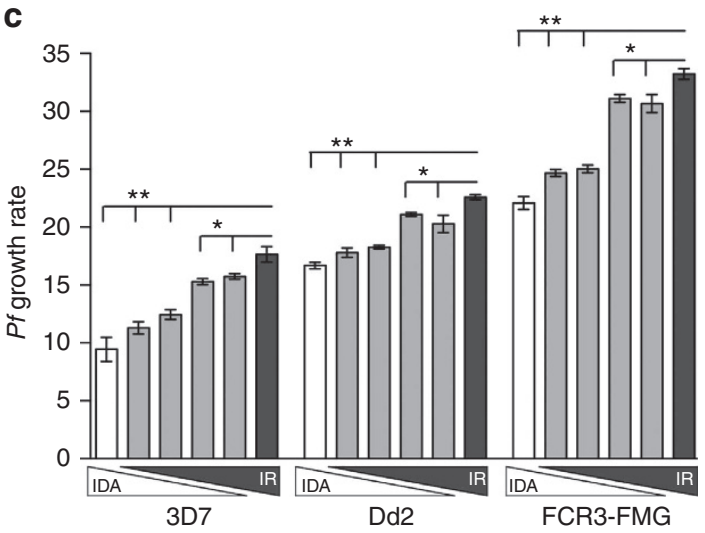

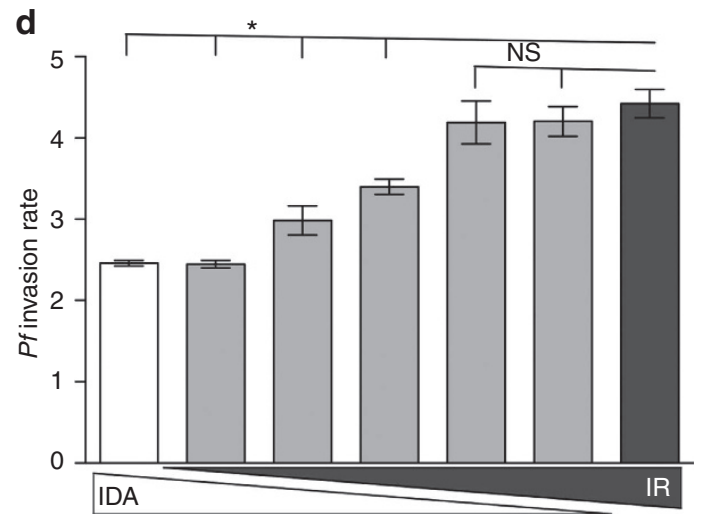

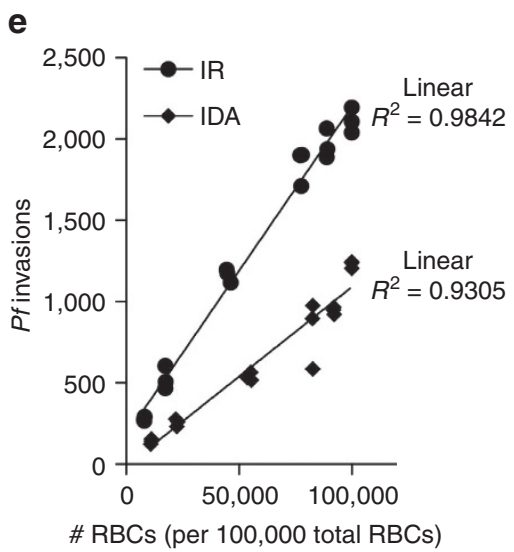

Figure 3 | Replacement of iron-deficient RBCs with iron-replete RBCs increases $\boldsymbol{P}$. falciparum infection. (a,b) P. falciparum (strain 3D7) infection of reticulocytes $(C D 71+)$ and mature $\mathrm{RBCs}(\mathrm{CD} 71-)$ from an IDA + Fe donor. (a) Bars represent the percent of parasitized reticulocytes (CD71 + ) and mature RBCs (CD71 - ). Error bars represent the s.d. ${ }^{\star} P<0.0001$. (b) Contribution of parasitized reticulocytes $(C D 71+)$ and parasitized mature RBCs $(C D 71-)$ to the total infection. Error bars represent the standard deviation. (c) Growth rate of $P$. falciparum in RBC populations in which IDA RBCs were replaced with IR RBCs. RBCs were inoculated individually or together in the same wells at different ratios (100\% IDA; $90 \%$ IDA and 10\% IR; 75\% IDA and $25 \%$ IR; $50 \%$ IDA and 50\% IR; $25 \%$ IDA and $75 \%$ IR; $100 \%$ IR) and subsequently infected. Elongated triangles below the $x$ axis represent the percentage of IDA RBCs (white triangle) and IR RBCs (grey triangle) in the total RBC population. Bars represent parasite growth rates after one $96 \mathrm{~h}$ growth assay. Error bars represent the s.d. ${ }^{\star} P<0.01$ and ${ }^{\star \star} P<0.0003$. (d and $\mathbf{e}$ ) Invasion rate of $P$. falciparum into RBC populations in which IDA RBCs were replaced with IR RBCs. Differentially labelled RBC donors were inoculated individually or together in the same wells at different ratios (100\% IDA; $90 \%$ IDA and 10\% IR; $80 \%$ IDA and $20 \%$ IR; $50 \%$ IDA and 50\% IR; $20 \%$ IDA and $80 \%$ IR; $10 \%$ IDA and $90 \%$ IR; $100 \%$ IR). Each invasion condition contained $20 \times 10^{6}$ total RBCs. (d) Bars represent parasite invasion rate. Elongated triangles below the $x$ axis represent the percentage of IDA RBCs (white triangle) and IR RBCs (grey triangle) in the total RBC population. Error bars represent the s.d. ${ }^{\star} P<0.001$, compared with $P$. falciparum invasion rate into $100 \%$ IR RBCs. (e) Number of invasions events into IDA (diamonds) and IR (circles) RBCs as the frequency of each increases. Linear regression was used to determine the best fit lines for the data (IR $R^{2}=0.9842$ and IDA $R^{2}=0.9305$ ). Analysis of covariance was performed to compare the slopes of the lines and calculated $P<0.0001$. The null hypothesis was no difference between the two RBC types $\left(H_{0}: \beta_{\text {Iron replete }}=\beta_{\text {Iron deficient, }} \alpha=0.05\right)$ n.s., nonsignificant.

invasion of RBCs from IDA donors to that of RBCs from IR donors. An SI of 1.0 indicates no difference in parasite invasion between two RBC populations. In experiments with strains 3D7, Dd2 and FCR3-FMG, the mean SI of RBCs from IDA donors relative to $\mathrm{RBCs}$ from $\mathrm{IR}$ donors was $0.56(95 \% \mathrm{CI}=0.56-0.57)$, $0.52(95 \% \quad \mathrm{CI}=0.52-0.53)$ and 0.70 (95\% $\mathrm{CI}=0.69-0.71)$, respectively, indicating consistently reduced invasion of RBCs from IDA donors (Fig. 2a). We next assessed parasite maturation within RBCs from IDA donors by analysing Giemsa-stained thing blood smears, which were made every $6 \mathrm{~h}$ during the course of a 48-h intra-erythrocytic lifecycle. We observed that parasites matured normally in iron-deficient RBCs, indicating that the reduced overall parasite growth in iron-deficient RBCs did not result from delayed maturation (Fig $2 b, c)$. Finally, we measured the parasitized erythrocyte multiplication rate (PEMR) ${ }^{17,18}$ of $P$. falciparum within RBCs from IDA donors as compared with RBCs from IR donors. For parasite strains 3D7, Dd2 and FCR3-
FMG, RBCs from IDA donors (relative to RBCs from IR donors) had a reduced PEMR of $48.0 \%$ (s.d. \pm 12.2 ), $25.7 \%$ (s.d. \pm 2.2 ) and $39.9 \%$ (s.d. \pm 9.3 ), respectively (Fig. 2 d). In accordance with the PEMR data, we additionally observed fewer merozoites within IDA as compared with IR RBCs by microscopy (data not shown). Taken together, these data indicate that $P$. falciparum matures normally within RBCs from IDA donors, but that invasion into and production of infectious merozoites within RBCs from IDA donors are significantly reduced. These data additionally reveal that different $P$. falciparum isolates may exhibit different invasion and PEMR phenotypes in RBCs of IDA individuals.

Replacement of iron-deficient RBCs increases malaria growth. The erythropoietic rate of iron-deficient individuals increases dramatically in response to iron supplementation, and elevated erythropoietic rate is hypothesized to increase an individual's 
susceptibility to malaria ${ }^{19}$. As the iron biomarkers of individuals in the IDA + Fe group were indicative of an erythropoietic response to iron (Table 1), we hypothesized that the replacement of iron-deficient RBCs with IR RBCs would explain the recovered growth of $P$. falciparum in RBCs from IDA + Fe donors (Fig. 1). To determine whether the replacement of iron-deficient RBCs with young IR RBCs could explain the recovered growth of $P$. falciparum in RBCs donated by IDA + Fe individuals, we first compared $P$. falciparum infection of reticulocytes $(C D 71+)$ and mature RBCs $(\mathrm{CD} 71-)$ from IDA + Fe donors. Consistent with $P$. falciparum's reported preference for young RBCs, we observed $8.6 \%$ (s.d. \pm 0.1$)$ parasitization of reticulocytes $(\mathrm{CD} 71+)$ and $4.5 \%$ (s.d. \pm 0.4 ) parasitization of mature RBCs (CD71-) (Fig. 3a). However, $(\mathrm{CD} 71+)$ reticulocytes accounted for only $1.6 \%$ (s.d. \pm 0.9 ) of all RBCs and parasitized $(\mathrm{CD} 71+)$ reticulocytes only contributed to $3.0 \%$ (s.d. \pm 0.1 ) of the total number of parasitized RBCs (Fig. 3b).

Having clearly demonstrated that reticulocytes from IDA $+\mathrm{Fe}$ donors are more highly infected by $P$. falciparum, but that reticulocytes themselves only contribute marginally to the total infection, we next sought to definitively determine whether the replacement of iron-deficient with IR RBCs could explain the recovered growth of $P$. falciparum in RBCs from $\mathrm{IDA}+\mathrm{Fe}$ donors. However, low incidence of IDA in our study setting, the difficulty of following iron-supplemented IDA individuals longitudinally through full recovery from iron deficiency, as well as the inability to use the common surrogates of RBC age (volume and density) against the background of changing host iron status, prevented us from studying the impact of an elevated erythropoietic rate on erythrocytic stage $P$. falciparum infection. Therefore, we modelled the effect of iron supplementation-mediated changes in RBC population dynamics on erythrocytic stage $P$. falciparum infection by assessing the impact of replacing RBCs from IDA donors with RBCs from IR donors on in vitro P. falciparum growth. Replacing $10,25,50$ and $75 \%$ of the RBCs from IDA donors with RBCs from IR donors resulted in a steady increase in parasite growth rate; $75 \%$ replacement recovered 3D7, Dd2 and FCR3-FMG $P$. falciparum growth rate to $88.2 \%$ (s.d. \pm 2.0 ), $89.7 \%$ (s.d. \pm 2.5 ) and $92.3 \%$ (s.d. \pm 1.1 ) that of the growth rate of in RBCs from IR donors, respectively (Fig. 3c).

As the first step in the erythrocytic life cycle of the malaria parasite, erythrocyte invasion is a pivotal determinant of the magnitude of infection. Therefore, we utilized the barcoded RBC invasion assay to determine how iron-deficient and IR RBCs interact to shape $P$. falciparum invasion. Like growth rate, $P$. falciparum invasion rate increased as IDA RBCs were replaced with IR RBCs. Rate of invasion was fully recovered to IR levels once $80 \%$ of IDA RBCs were replaced with IR RBCs for all three parasite strains (Fig. 3d and Supplementary Fig. 2a). Furthermore, employment of the barcoded RBC invasion assay allowed for the full characterization of the kinetics of $P$. falciparum invasion into IDA and IR RBCs, as the frequency of each changed relative to the total RBC population. We observed that the number of $P$. falciparum FCR3-FMG invasions into IDA and IR RBCs increased linearly, as each RBC population increased in frequency, $R^{2}=0.984$ and 0.931 , respectively, and $P$. falciparum invasion as a function of IDA RBC abundance was significantly less than that of IR RBCs (Fig. 3e). Similar trends were observed for P. falciparum strains 3D7 and Dd2 (Supplementary Fig. 2b and c). Together, these results support the hypothesis that replacing an individual's iron-deficient RBC population with IR RBCs would increase the host's susceptibility to erythrocytic stage $P$. falciparum infection and clearly illustrate the impact $\mathrm{RBC}$ population dynamics have on potential parasite biomass and pathogenesis. Moreover, our use of the barcoded RBC invasion assay allowed for the definitive determination and characterization of the distribution of parasites in IDA and IR RBC populations, as the frequency of each changed.

Effect of RBC population age structure on malaria infection. Reticulocytes and young RBCs are preferentially invaded by P. falciparum ${ }^{20,21}$, and theoretical models predict that elevated reticulocytosis may increase the risk of high parasite density ${ }^{19,22}$. Because of the shortened lifespan of iron-deficient RBCs, there is a period in the course of an individual's recovery from IDA at which point protective iron-deficient RBCs have been cleared from circulation and the remaining circulating RBCs are on average younger than that of an IR individual. To determine the capacity of young RBCs to shape P. falciparum infection, we (i) directly compare P. falciparum infection of young RBCs and RBCs of increasing age and (ii) model the effect of replacing young IR RBCs with old IR RBCs on $P$. falciparum growth and invasion in vitro. For these studies, we utilized two proxies for $\mathrm{RBC}$ age: $\mathrm{RBC}$ volume, which decreases with age ${ }^{23}$ and is unaffected by ring stage parasitization ${ }^{24}$, and $\mathrm{RBC}$ density, which increases with increasing $\mathrm{RBC}$ age ${ }^{23}$. We observed that when RBCs from an IR donor were infected with P. falciparum, parasite infection increased with increasing RBC volume (Fig. 4a). To directly compare $P$. falciparum invasion of RBCs of different ages, we density separated RBCs from IR donors into four fractions of increasing RBC age: young, young adult, mature adult and old (Supplementary Fig. 3A). Decreasing MCV, reticulocyte content and Calcein fluorescence ${ }^{25}$ confirmed the age separation of the RBCs (Supplementary Fig. 3b-d). We observed that the SI of young adult, mature adult and old RBCs to parasite invasion as compared with young RBCs was $0.85(95 \% \mathrm{CI}=0.82-0.90), 0.58$ $(95 \% \mathrm{CI}=0.56-0.62)$ and $0.28(95 \% \mathrm{CI}=0.27-0.30)$, respectively (Fig. 4b). In accordance with previous reports ${ }^{17,20,26}$, young RBCs sustained a significantly greater growth rate than young adult, mature adult and old RBCs, with young RBCs supporting a growth rate $50 \%$ greater than old RBCs (Supplementary Fig. 3e), and compared with young RBCs the PEMR was reduced by $10 \%$ (s.d. \pm 4.78 ), 15\% (s.d. \pm 1.16 ) and 19\% (s.d. \pm 2.23 ) in young adult, mature adult and old RBCs, respectively (Supplementary Fig. 3f). These data clearly demonstrate the preferential invasion of $P$. falciparum into young RBCs and show that the risk of RBCs to $P$. falciparum invasion relative to young RBCs decreases with increasing $\mathrm{RBC}$ age. All together we have confirmed (i) $P$. falciparum infection is more prevalent in young RBCs (ii) the increased capacity of young RBCs to support $P$. falciparum invasion and growth.

To determine the capacity of the age distribution of a RBC population to shape $P$. falciparum infection, we examined the effect of replacing young IR RBCs with old IR RBCs on P. falciparum growth in vitro. We observed $15.7 \%$ (s.d. \pm 3.1 ) greater growth of $P$. falciparum in young IR RBCs as compared with density separated and then recombined (total) IR RBCs, and growth remained significantly greater when $10 \%$ of young RBCs were replaced with old RBCs. Following replacement of 33, 50, 66, 80 and $90 \%$ of young RBCs with old RBCs, P. falciparum growth rate steadily decreased (Fig. 4c). Consistent with $P$. falciparum growth, we observe that the rate of $P$. falciparum invasion into young RBCs is significantly greater than that of total IR RBCs $(29.0 \%$ (s.d. \pm 2.1$))$. Furthermore, we observed that as the frequency of young RBCs decreased from 100 to $50 \%$ and the frequency of old RBCs increased from 0 to $50 \%$, the total rate of invasion decreased by only $4.9 \%$ (s.d. \pm 0.7 ), maintaining a $P$. falciparum invasion rate significantly greater than that of the invasion rate of total RBCs. However, when the frequency of young RBCs fell from 50 to $0 \%$ and old RBCs increased from 50 
a

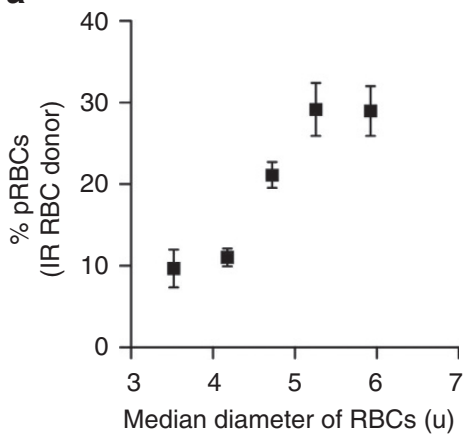

b

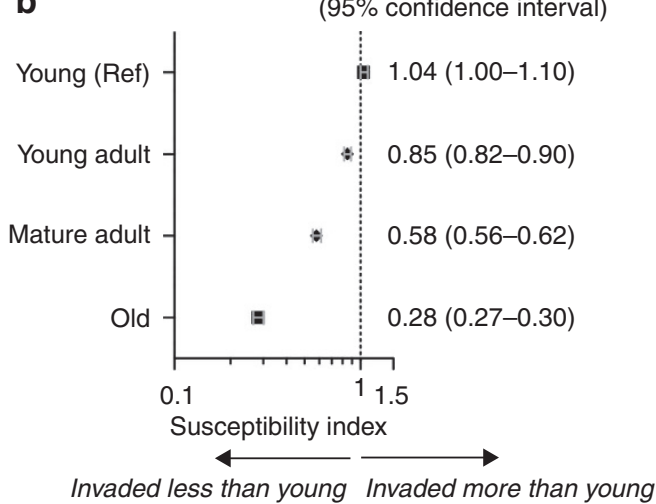

C

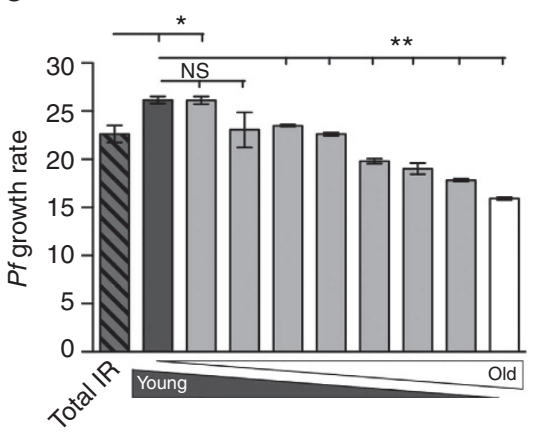

d

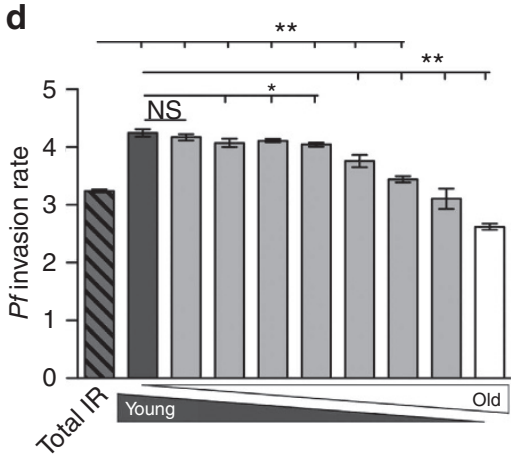

e

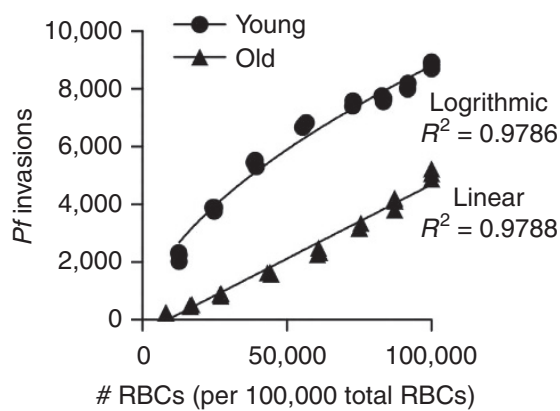

Figure 4 | The elevated $\boldsymbol{P}$. falciparum infection supported by young RBCs is reversed as young RBCs are replaced with old RBCs. (a) Percent $P$. falciparum (strain 3D7) infection of RBCs of increasing diameter. Data points represent the \% pRBCs of five gated RBC populations of increasing volume. Error bars represent the s.d. (b) Direct comparison of $P$. falciparum (strain FCR3-FMG) invasion into RBCs of increasing age. IR RBCs were separated into five fractions of increasing density, a proxy for increasing RBC age (Supplementary Fig. 3a-d). The markers represent the SI point estimate and the bar represents the $95 \% \mathrm{Cl}$. (c) Growth rate of $P$. falciparum (strain FCR3-FMG) in RBC populations in which young IR RBCs were replaced with old IR RBCs (0\%, 10\%, 20\%,33\%, 50\%, 66\%, 80\%, 90\% and 100\% replacement young RBCs with old RBCs). Elongated triangles represent the percentage of young IR RBCs (gray triangle) and old IR RBCs (white triangle) in the total RBC population. Bars represent parasite growth rates after $96 \mathrm{~h}$ Error bars represent the s.d. ${ }^{\star} P<0.004$ and ${ }^{\star \star} P<0.0003$, compared to growth rate in $100 \%$ total IR and $100 \%$ young RBCs respectively. (d and $\mathbf{e}$ ) Invasion into RBC populations in which young IR RBCs were replaced with old IR RBCs. Differentially labelled young and old RBCs were inoculated individually or together in the same wells at different ratios (0\%, 10\%, 20\%, 33\%, 50\%, 66\%, 80\%, 90\% and 100\% replacement young RBCs with old RBCs). (d) Bars represent invasion rates. Elongated triangles represent the percentage of young IR RBCs (gray triangle) and old IR RBCs (white triangle) in the total RBC population. Error bars represent the s.d. ${ }^{\star} P<0.05$ and ${ }^{\star *} P<0.003$ (e) Number of invasions events into young (circles) and old (triangles) RBCs as the frequency of each increases. Linear regression was used to determine best fit lines. A linear function best fit old RBC data $\left(R^{2}=0.9788\right)$ and a logarithmic function best fit young RBC data $\left(R^{2}=0.9786\right)$. Analysis of covariance was performed to determine whether invasion data of old and young RBCs differed significantly, $P<0.0001$. The null hypothesis was no difference between the two RBC types $\left(H_{0}: \beta_{\text {Iron replete }}=\beta_{\text {Iron deficient, }} \alpha=0.05\right)$ n.s., nonsignificant.

to $100 \%, P$. falciparum infection decreased steadily, ultimately falling by $45.7 \%$ (s.d. \pm 1.9 ) (Fig. $4 \mathrm{~d}$ ).

As young IR RBCs are at the greatest risk of $P$. falciparum invasion, we speculated that an insufficient merozoite inoculum might be responsible for the observed plateau in P. falciparum invasion. However, invasion experiments with double the inoculum of merozoites also resulted in a plateau in the rate of $P$. falciparum invasion when young RBCs accounted for more than $50 \%$ of the total RBCs population (Supplementary Fig. 4a). Moreover, the rate of $P$. falciparum invasion achieved with the higher inoculum was less than that of the lower inoculum invasion experiments (Fig. 4d and Supplementary Fig. 4a). We subsequently characterized the kinetics of $P$. falciparum invasion into young and old RBCs as the frequency of each changed relative to the total $\mathrm{RBC}$ population. We observed that like $P$. falciparum invasion of IDA and IR RBCs, the number of $P$. falciparum invasions into old IR RBCs increased linearly as old IR RBCs increased in frequency, $R^{2}=0.991$. In contrast, the number of $P$. falciparum invasions into young IR RBCs as a function of young IR RBC abundance was best fit by a logarithmic function, $R^{2}=0.976$ (Fig. 4e). The same kinetics of $P$. falciparum invasion were observed when experiments were performed with double the merozoite inoculum (Supplementary Fig. 4b). These results demonstrate that replacement of young IR RBCs with old IR RBCs reverses the elevated growth and invasion rate sustained by young IR RBCs. In addition, we show that the rate of $P$. falciparum invasion only begins to dramatically drop off once $50 \%$ of young IR RBCs have been replaced with old IR RBCs, and that this can be attributed to the logarithmic nature of $P$. falciparum invasion of young IR RBCs. Together, these data support the hypothesis that the effects of iron deficiency and iron supplementation on RBC physiology and erythropoietic rate are at least partially responsible for determining an individual's risk of malaria infection (Fig. 5).

\section{Discussion}

Iron supplementation has clear nutritional benefits for children and pregnant women ${ }^{2}$, but iron is also an essential nutrient for most pathogens and as a result is a critical mediator of 
host-pathogen interactions ${ }^{27}$. Activation of the host innate immune system by the malaria parasite or other infectious organisms triggers reduction in iron absorption, redistribution of existing iron stores and decreases erythropoiesis, which effectively limits the availability of iron to invading pathogens. It is unknown what host iron (mosquito or human) P. falciparum is able to access and utilize nor how the parasite circumvents the host's attempt to restrict iron. It has been previously postulated that as occurs with other pathogens ${ }^{28}$, iron deficiency inhibits $P$. falciparum infection via iron deprivation. Although the malaria parasite may find iron less readily available in an iron-deficient host, our work reveals an alternate cellular mechanism by which iron deficiency may protect against malaria. Our study of the relationship between iron deficiency, iron supplementation and erythrocytic stage $P$. falciparum infection highlights how by altering the dynamics of the human hosts RBC population iron deficiency and iron supplementation shape erythrocytic stage $P$. falciparum infection.

Clinical studies in different field sites have reported that iron deficiency correlates with protection from malaria. In Malawian children, baseline iron deficiency was associated with significant reductions in the subsequent risks of both parasitemia (45\%) and malaria $(51 \%)^{29}$. Similarly, in Tanzanian children, baseline iron deficiency significantly decreased the odds of subsequent parasitemia $(23 \%)$ and severe malaria $(38 \%)^{6}$. In addition, in two studies of pregnant women, iron deficiency was associated with a decreased prevalence of placental malaria, a major cause of neonatal and maternal morbidity ${ }^{3,4}$. Our results-that irondeficient RBCs impair parasite propagation in vitro (Fig. 1)-are consistent with these clinical findings, and provide valuable insight into a cellular mechanism for the observations made in the clinical setting. In our study of $P$. falciparum growth in RBCs from IDA donors, we reveal that RBCs from IDA donors are refractory to $P$. falciparum invasion and support a lower PEMR but that parasite maturation is normal (Fig. 2). There are multiple physiological differences between iron-deficient and IR RBCs that may contribute to the impaired invasion into and replication within iron-deficient RBCs. These include greater osmotic fragility and membrane rigidity, accelerated ageing in vivo ${ }^{30-33}$, lower Hgb content and smaller size (microcytosis).

Iron supplementation has long been hypothesized to increase malaria risk, and this issue has garnered recent attention after a large-scale nutritional supplementation study in Tanzanian children was halted owing to significantly increased mortality among those receiving iron ${ }^{8}$. Although it remains unclear whether the increased mortality rate was secondary to malaria, this potential for harm has complicated recommendations for widespread supplementation and has caused iron supplementation programmes in malaria endemic countries to be suspended. In a more recent randomized trial of Tanzanian children, iron supplementation increased the risk of malaria by $41 \%$ in iron-deficient but not in IR children ${ }^{8}$. Notably, our studies are consistent with the Tanzania study. Specifically, we observed increased parasite growth in RBCs donated by iron-supplemented IDA individuals and a modest effect in RBCs donated by iron-supplemented IR individuals (Fig. 1). As individuals in the iron-supplemented IDA group were observed to be undergoing an erythropoietic response to iron supplementation (Table 1), we proceeded to investigate the effect of replacing iron-deficient

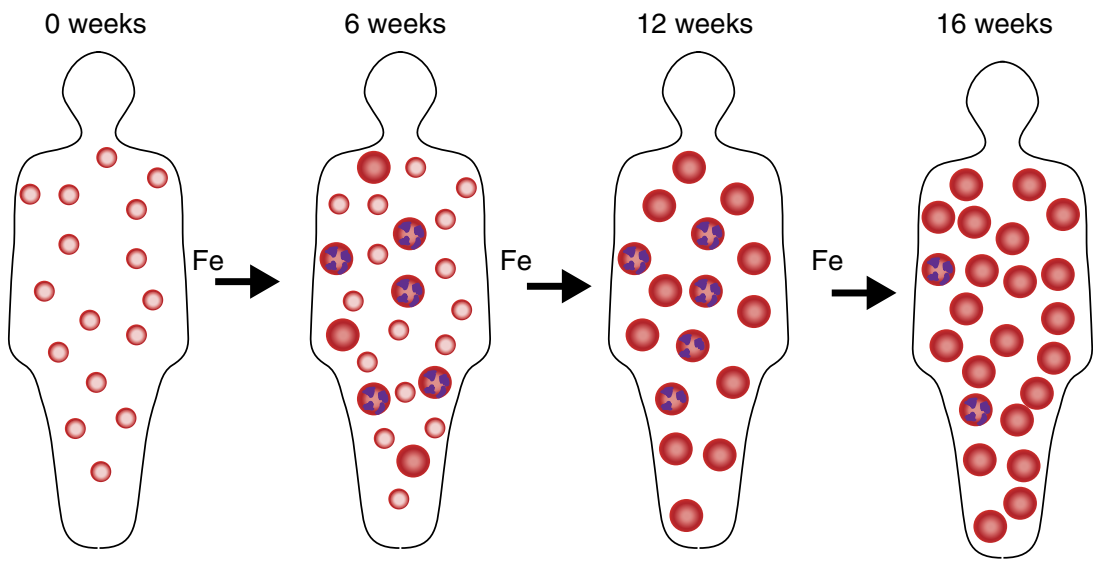

Relative susceptibility to malaria

Microcytic, iron-deficient RBC
Reticulocyte
Normal RBC

Figure 5 | Hypothesized impact of iron deficiency and iron supplementation on host RBC population dynamics and susceptibility to erythrocytic stage malaria infection. Recovery from IDA is a complex process, which varies between individuals. Iron supplementation of an individual with IDA (0 weeks) will result in reticulocytosis and the production of young iron-replete RBCs ( 6 weeks). 12 weeks after the initiation of supplementation, the majority of the irondeficient RBCs, will have been cleared from circulation (iron-deficient and iron-replete RBCs have 90 and 120 day lifespans respectively). After 16 weeks of iron supplementation, iron status has been corrected and the age structure of the RBC population will be restored. As shown above, we hypothesize that individuals with IDA will be less susceptible to erythrocytic stage malaria. The induction of erythropoiesis in these individual by iron supplementation and subsequent replacement of the iron-deficient RBCs with young iron-replete RBCs will increase the susceptibility of the individual to erythrocytic stage malaria infection. The susceptibility to infection is predicted to peak at the point when all iron-deficient RBCs have been replaced, but the age distribution of iron-replete RBCs is on average younger than a fully recovered iron-replete individual. Finally, restoration of the normal distribution of RBC age will return an individual's susceptibility to a normal level. 
RBCs with IR RBCs and young RBCs with old RBCs on $P$. falciparum erythrocyte infection. We demonstrate that $P$. falciparum growth and invasion rates increase when irondeficient RBCs are replaced with IR RBCs (Fig. 3). Furthermore, we show that young RBCs support greater $P$. falciparum growth and invasion rates than total IR RBCs and that the replacement of young RBCs with old RBCs reverses the effect of young RBCs on parasite growth and invasion (Fig. 4). Finally, in the course of these experiments, our use of barcoded RBC flow cytometry invasion assay has provided novel insight into the relationship between the frequency of a RBC subset in the total RBC population and P. falciparum infection (Figs $3 e$ and 4e). In summary, our results demonstrate that the changes in the RBC population that occur during recovery from IDA enhance parasite propagation.

It should be noted that our work exclusively focuses on the influence of iron deficiency and iron supplementation on the susceptibility of the human host's RBC to malaria infection, and does not address the potential effect of serum iron ${ }^{13}$ or additional factors, which may function in vivo, including growth of the hepatic stage of the parasite ${ }^{34}$, rosetting and cytoadherence to the endothelium, accelerated clearance of parasitized $\mathrm{RBCs}^{35,36}$, effects of innate immune factors such as hepcidin ${ }^{11}$ and lipocalin 2 (ref. 37), or adaptive immune function. We have previously reported that both transferrin and ferric citrate increase the bioavailable iron pool of trophozoite-infected RBCs but not that of uninfected $\mathrm{RBCs}^{13}$. It is possible that parasite growth may be enhanced by the transient increase in serum iron that is observed in IR individuals who are given oral iron supplementation ${ }^{12}$. Further investigations need to be conducted to explore the extent to which iron deficiency and iron supplementation shape other aspects of malaria pathogenesis.

Our findings, taken together with those from field studies, raise the important medical and public health question: How can iron supplementation be safely administered to IDA children in malarious areas? A critical implication of these observations is that reconstitution of red cell mass in anaemic patients would be expected to transiently enhance susceptibility to malaria (Fig. 5), which may inform the on-going debate as to whether fortification with iron would be safer than supplemental iron. Our data implies that, where $P$. falciparum is endemic, treatment of anaemia with iron supplementation should be accompanied by malaria preventive measures, such as malaria prophylaxis, bed nets and increased active surveillance and access to health care. Additional questions raised by this study are: Does iron deficiency in African children represent an evolutionarily advantageous phenotype that derives from polymorphisms in iron homeostasis? What molecular mechanisms confer protection from malaria in the setting of microcytosis, and can these protective mechanisms be exploited by medical interventions? Future clinical and translational studies will be needed in order to design safe and effective interventions to address the twin burdens of iron deficiency and falciparum malaria.

\section{Methods}

Clinical. Study participants included healthy, HIV-negative, non-pregnant donors over 18 years of age with and without IDA. Exclusion criteria included: on-going inflammation or infection, previous history of malaria, travel to malaria endemic areas, malignancy, sickle cell disease (or trait), thalassemia (or trait for either thalassemia- $\alpha$ or $-\beta$ ). This study was approved by the University of North Carolina Institutional Review Board, Protocol \# 09-0559, and informed consent was obtained from all subjects. Study participants with $\mathrm{Hgb}>11 \mathrm{~g} \mathrm{dl}^{-1}$ and ferritin $>12 \mathrm{ng} \mathrm{ml}^{-1}$ were classified as IR and participants with $\mathrm{Hgb}<11 \mathrm{~g} \mathrm{dl}^{-1}$ and ferritin $<12 \mathrm{ng} \mathrm{ml}^{-1}$ were classified as IDA. IDA + Fe donors were identified by their personal physicians for participation in our study. Subjects were included in the IDA + Fe group if they fit the criteria for IDA and had been prescribed highdose oral ferrous sulfate, $60 \mathrm{mg}\left(9-12.6 \mathrm{mg} \mathrm{kg}^{-1}\right)$ elemental iron orally three times per day or intravenous iron at a dosage determined by their personal physician using the following equation: Dose $=0.0442$ [desired Hgb - observed Hgb] $\times$ LBW $+[0.26 \times$ LBW] . Healthy donors took $325 \mathrm{mg}$ of ferrous sulfate once daily for the 2-month duration of the study. These donors donated $40 \mathrm{ml}$ of blood on three occasions - at enrollment and two subsequent monthly intervals. An additional group of healthy donors served as the IR control group. Non-anaemic donors with low-iron stores $\left(\mathrm{Hgb}>11 \mathrm{~g} \mathrm{dl}^{-1}\right.$, ferritin $<12 \mathrm{ng} \mathrm{ml}^{-1}$ ) were excluded. Full iron panels were obtained for each study participant and mean values for each group are reported in Table 1.

Parasite culture. P. falciparum parasite strains 3D7, Dd2 and FCR3-FMG were cultured in RBCs from O+, IR donors at 2-3\% haematocrit and Albumax Complete Media (ACM)-RPMI 1640 (Sigma-Aldrich) with 10\% AlbuMAX II (Gibco), $1 \mathrm{mM}$ hypoxanthine (Sigma-Aldrich), $20 \mathrm{mM}$ L-glutamine (Cellgro), $0.45 \%$ glucose (Cellgro) and $0.01 \mathrm{ng} \mathrm{ml}^{-1}$ gentamicin (Sigma-Aldrich). AlbuMAX II was used to supplement the media in place of human serum to isolate the effects of the RBCs from different experimental groups. All RBCs used for parasite culture were obtained from well-characterized IR O + donors and used within 14 days of being drawn. Cultured parasites were maintained between 0.5 and $10 \%$ at $37^{\circ} \mathrm{C}$, in an atmosphere of $5 \% \mathrm{O}_{2}, 5 \% \mathrm{CO}_{2}$ and $90 \% \mathrm{~N}_{2}$ with continuous shaking. Early ring stage parasites were synchronized with $5 \%(\mathrm{w} / \mathrm{v}) \mathrm{D}$-sorbitol. Synchronization was repeated at 20 and $40 \mathrm{~h}$ to achieve a tightly synchronized parasite population.

Growth assay. P. falciparum parasites from routine cultures were seeded as rings at $0.5 \%$ initial parasitemia in $1 \%$ haematocrit in ACM in triplicate in 96-well plates Parasites were maintained for $96 \mathrm{~h}$ under standard culture conditions and the media was changed daily. At $96 \mathrm{~h}$, parasite cultures were split back to $0.5 \%$ parasitemia and maintained as described for an additional $96 \mathrm{~h}$ (Supplementary Fig. 1). Parasites were stained at all 0 and $96 \mathrm{~h}$ time points with $1 \times$ DNA dye SYBR Green I (Invitrogen) as described in ref. 38, and fixed in 1\% paraformaldehyde and $0.0075 \%$ glutaraldehyde (Electron Microscopy Sciences) in Alsever's Solution (Sigma-Aldrich) for $30 \mathrm{~min}$ at $4^{\circ} \mathrm{C}$. Fixative was removed and cells were stored in PBS at $4^{\circ} \mathrm{C}$ until analysis by flow cytometry. Growth rate reflects the fold increase in RBC parasitization between 0 and $96 \mathrm{~h}$. To identify parasitized reticulocytes, cultures were stained with $0.5 \mu \mathrm{M}$ DNA dye SYTO 61 (Invitrogen) ${ }^{13,39}$ and PEconjugated mouse anti-human CD71 antibody (Miltenyi Biotech) and analysed by flow cytometry.

Barcoded RBC invasion assay. RBCs were labelled with $5 \mu \mathrm{M}$ of either CellTrace Violet or CellTrace Far Red DDAO-SE (Invitrogen) as described ${ }^{39}$. Violet- and DDAO-labelled RBCs were combined in ACM and delivered in triplicate into 96well plates and subsequently seeded with schizonts to achieve $1.5-2 \%$ parasitized RBCs. Parasites were maintained for $12-18 \mathrm{~h}$ under standard culture conditions to allow for schizont rupture and subsequent invasion of CellTrace-labelled RBCs. The invasion of $P$. falciparum into Violet- and DDAO-labelled RBCs was directly compared by measuring the SI, defined as the ratio of the prevalences of infected Violet RBCs to infected DDAO RBCs ${ }^{40}$. Note that the SI is different from the previously described selectivity index ${ }^{18,41}$. The selectivity index has been used to report the number of multiply infected cells, whereas the SI defines the relative susceptibility to invasion of two different types of RBCs.

Parasite erythrocyte multiplication rate assay. Trophozoite-infected cultures were magnetic activated cell sorting (MACS) purified and seeded into experimental RBCs to achieve $1.5-2 \%$ pRBCs and incubated for $48 \mathrm{~h}$ to allow for invasion of merozoites into experimental RBCs and their subsequent development into trophozoites inside the experimental RBCs. Experimental RBC parasite density was determined and the same number of infected experimental RBCs was seeded into separate wells containing target RBCs (from an IR donor) to achieve $1.5-2 \%$ pRBCs. Cultures were then incubated for $12-18 \mathrm{~h}$ to allow merozoites produced within experimental RBCs to invade target RBCs, allowing for assessment of the $\mathrm{PEMR}^{17,18}$. Following invasion of target RBCs, cells were stained with SYBR Green I and analysed by flow cytometry to determine the total number of ring pRBCs. PEMR reflects the number of merozoite invasions of target RBCs per schizont infected experimental RBC.

Density separation. RBCs were separated into five fractions with a modified version of previously described density gradient centrifugation method ${ }^{23}$. Briefly, blood was collected into acid citrate dextrose and plasma was subsequently removed by centrifugation for $15 \mathrm{~min}$ at $800 \mathrm{~g}$. Packed cells were resuspended at $50 \%$ haematocrit in RPMI, and passed over a $2: 1(\mathrm{w} / \mathrm{w}) \alpha$-cellulose/microcrystalline cellulose column to remove lymphocytes ${ }^{42}$. Following lymphocyte depletion, RBCs were washed twice with RBC buffer (10 mM HEPES, $12 \mathrm{mM} \mathrm{NaCl}, 115 \mathrm{mM} \mathrm{KCl}$ $5 \%$ BSA). RBCs were layered onto a 65, 60, 55 and 50\% discontinuous Percoll gradient and then centrifuged for $25 \mathrm{~min}$ at $1,075 \mathrm{~g}$. Each of the five fractions was removed, washed twice with $\mathrm{RBC}$ buffer and stored at $4{ }^{\circ} \mathrm{C}$ for up to 5 days. Decreasing MCV, reticulocyte content and Calcein fluorescence ${ }^{25}$ of the five fractions confirmed the age separation of RBCs (Supplementary Fig. 3c-g). For barcoded RBC invasion assays and growth assays, which utilized density separated RBCs, recombined RBCs were included as a 'total' RBC control. 
Flow cytometry. Growth, invasion and infectious merozoite production assays were analysed by flow cytometry using either a modified FACS-Calibur with two lasers $30 \mathrm{~mW} 488$ Diode Pumped Solid State laser and a 25-mW 637 red diode laser (FACS-Calibur; Becton Dickinson, modified by Cytek Development) or a Beckman-Coulter (Dako) CyAn ADP. Channels and probes used on the FACSCalibur included: SYTO 61 (637 nM, 666/27 bandpass), PE (488 nM, 585/42 bandpass) and SYBR Green I ( $488 \mathrm{nM}, 530 / 30$ bandpass). FACS-Calibur data were collected using FlowJo CE and analysed with Summit v5.1. Channels and probes used on the Dako cyan included: CellTrace Violet ( $405 \mathrm{nM}, 450 / 50$ bandpass), SYBR Green I ( $488 \mathrm{nM}, 530 / 40$ bandpass) and CellTrace DDAO-SE (635 nM, $665 / 20$ bandpass). Data from the Cyan cyotometer were collected and analysed with Summit v4.3.01. Linear amplification of forward scatter was used to set event threshold in order to exclude cell debris, microparticles and doublets. Electronic volume of uninfected RBCs and pRBCs was assessed on a Beckman Coulter Cell lab Quanta (Beckman Coulter). Channels and probes used on the Quanta included: SYBR Green I ( $488 \mathrm{nM}, 525 / 40$ bandpass). National Institute of Standards and Technologies-certified beads standard L2 $2 \mu \mathrm{m}$, L5 $5 \mu \mathrm{m}$ and L10 $10 \mu \mathrm{m}$ (Beckman Coulter) were used to calibrate electronic volume. Calibrated data were expressed as both electronic volume $\left(\mu \mathrm{m}^{3}\right)$ and diameter $(\mu \mathrm{m})$. Quanta data were collected with Cell Lab Quanta Collection Software for Instrument Control and analysed with Kaluza (Beckman Coulter). For all experiments, samples were diluted to $0.001-0.002 \%$ haematocrit and 100,000-500,000 total events were collected.

Statistical methods. All experiments were performed in triplicate. Results are from either one representative experiment or the combined results of at least three independent experiments. Parasite growth rate and infectious merozoite production experiments were analysed with two-tailed Student's $t$-test and one-way analysis of variance (GraphPad Prism 5).

Data from all in vitro growth studies were pooled and analysed using random effects regression (Fig. 1d,e). The dependent variable in the analysis was the logarithm of the ratio of the percent $\mathrm{pRBCs}$ at $96 \mathrm{~h}$ (final) and $0 \mathrm{~h}$ (initial). In addition, to the usual variation independently affecting each observation, we fitted two higher levels of variance: variation between individuals and day-to-day variation in parasite 'preparations' taking account of the fact that in this data set these two variance components were cross (rather than the more usually encountered nested design). We fitted two exposure variables, iron status and iron supplementation, both as binary variables, and their interaction, focusing on three contrasts: supplemented IDA versus supplemented IR donors; supplemented versus non-supplemented IDA donors; supplemented versus non-supplemented IR donors. All experiments were performed in triplicate with three $P$. falciparum strains (3D7, Dd2 and FCR3-FMG) and consisted of three serial $96 \mathrm{~h}$ growth assays (Supplementary Fig. 1); P. falciparum strain, growth assay number and their (highly significant) interaction were fitted as binary covariates. We noted that when $0 \mathrm{~h}$ (initial) percent $\mathrm{pRBCs}$ was greater than 1 , the second growth assay always gave anomalous low results (most likely due to a saturation effect). As these data were uninformative and yet added noise to the analysis, we omitted all such cases while noting that although their inclusion increased the standard errors, it did not change the same general conclusions of the analysis. This model was fitted using Stata's xtmixed procedure (v12, Stata Corp).

To compare the susceptibility of different RBC types to invasion by P. falciparum, an unadjusted odds ratio was used to calculate the SI. All statistical analyses for invasion experiments were performed with Stata/IC (v10, Stata Corp). Linear regression was employed to investigate the associations between host iron status and parasite invasion in vitro using percent of RBCs from IDA or IR donors as the independent variable and total invasions $/ 1 \times 10^{5} \mathrm{RBCs}$ as the dependent variable (Fig. 3e). Analysis of covariance was conducted to determine whether the invasion into the two groups were the same. An $\alpha$ of 0.05 was set a priori to determine statistically significant differences. Similar analysis was done to better understand associations between RBC age and parasite invasion (Fig. 4e).

\section{References}

1. Price, R. N. et al. Factors contributing to anemia after uncomplicated falciparum malaria. Am. J. Trop. Med. Hyg. 65, 614-622 (2001).

2. Nweneka, C. V., Doherty, C. P., Cox, S. \& Prentice, A. Iron delocalisation in the pathogenesis of malarial anaemia. Trans. R. Soc. Trop. Med. Hyg. 104, 175-184 (2010).

3. Kabyemela, E. R., Fried, M., Kurtis, J. D., Mutabingwa, T. K. \& Duffy, P. E. Decreased susceptibility to Plasmodium falciparum infection in pregnant women with iron deficiency. J. Infect. Dis. 198, 163-166 (2008).

4. Senga, E. L., Harper, G., Koshy, G., Kazembe, P. N. \& Brabin, B. J. Reduced risk for placental malaria in iron deficient women. Malar. J. 10, 47 (2011).

5. Sangaré, L., van Eijk, A. M., Ter Kuile, F. O., Walson, J. \& Stergachis, A. The association between malaria and iron status or supplementation in pregnancy: a systematic review and meta-analysis. PLoS ONE 9, e87743 (2014).

6. Gwamaka, M. et al. Iron deficiency protects against severe Plasmodium falciparum malaria and death in young children. Clin. Infect. Dis. 54, 1137-1144 (2012).

7. Nyakeriga, A. M. et al. Iron deficiency and malaria among children living on the coast of Kenya. J. Infect. Dis. 190, 439-447 (2004).
8. Veenemans, J. et al. Effect of supplementation with zinc and other micronutrients on malaria in Tanzanian children: a randomised trial. PLoS Med. 8, e1001125 (2011).

9. Sazawal, S. et al. Effects of routine prophylactic supplementation with iron and folic acid on admission to hospital and mortality in preschool children in a high malaria transmission setting: community-based, randomised, placebo-controlled trial. Lancet 367, 133-143 (2006).

10. Soofi, S. et al. Effect of provision of daily zinc and iron with several micronutrients on growth and morbidity among young children in Pakistan: a cluster-randomised trial. Lancet 382, 29-40 (2013).

11. Portugal, S. et al. Host-mediated regulation of superinfection in malaria. Nat. Med 17, 732-737 (2011).

12. Schümann, K. et al. Impact of oral iron challenges on circulating nontransferrin-bound iron in healthy Guatemalan males. Ann. Nutr. Metab. 60, 98-107 (2012).

13. Clark, M., Fisher, N. C., Kasthuri, R. \& Cerami Hand, C. Parasite maturation and host serum iron influence the labile iron pool of erythrocyte stage Plasmodium falciparum. Br. J. Haematol. 161, 262-269 (2013).

14. Bronzan, R. N. et al. Bacteremia in Malawian children with severe malaria: prevalence, etiology, HIV coinfection, and outcome. J. Infect. Dis. 195, 895-904 (2007).

15. Berkley, J. A. et al. HIV infection, malnutrition, and invasive bacterial infection among children with severe malaria. Clin. Infect. Dis. 49, 336-343 (2009).

16. Church, J. \& Maitland, K. Invasive bacterial co-infection in African children with Plasmodium falciparum malaria: a systematic review. BMC. Med. 12, 31 (2014).

17. Lim, C. et al. Expansion of host cellular niche can drive adaptation of a zoonotic malaria parasite to humans. Nat. Commun. 4, 1638 (2013).

18. Chotivanich, K. et al. Parasite multiplication potential and the severity of falciparum malaria. J. Infect. Dis. 181, 1206-1209 (2000).

19. Cromer, D., Stark, J. \& Davenport, M. P. Low red cell production may protect against severe anemia during a malaria infection--insights from modeling. J. Theor. Biol. 257, 533-542 (2009).

20. Pasvol, G., Weatherall, D. J. \& Wilson, R. J. The increased susceptibility of young red cells to invasion by the malarial parasite Plasmodium falciparum. $\mathrm{Br}$ J. Haematol. 45, 285-295 (1980).

21. Tian, L. P. et al. Red cell age and susceptibility to malaria during pregnancy. Acta. Obstet. Gynecol. Scand. 77, 717-721 (1998).

22. McQueen, P. G. \& McKenzie, F. E. Age-structured red blood cell susceptibility and the dynamics of malaria infections. Proc. Natl Acad. Sci. USA 101, 9161-9166 (2004).

23. Bosch, F. H. et al. Characteristics of red blood cell populations fractionated with a combination of counterflow centrifugation and Percoll separation. Blood 79, 254-260 (1992).

24. Esposito, A. et al. Quantitative imaging of human red blood cells infected with Plasmodium falciparum. Biophys. J. 99, 953-960 (2010).

25. Bratosin, D., Mitrofan, L., Palii, C., Estaquier, J. \& Montreuil, J. Novel fluorescence assay using calcein-AM for the determination of human erythrocyte viability and aging. Cytometry A 66, 78-84 (2005).

26. Tiffert, T. et al. The hydration state of human red blood cells and their susceptibility to invasion by Plasmodium falciparum. Blood 105, 4853-4860 (2005).

27. Drakesmith, H. \& Prentice, A. M. Hepcidin and the iron-infection axis. Science 338, 768-772 (2012).

28. Skaar, E. P. The battle for iron between bacterial pathogens and their vertebrate hosts. PLoS Pathog. 6, e1000949 (2010).

29. Jonker, F. A. M. et al. Iron status predicts malaria risk in Malawian preschool children. PLoS ONE 7, e42670 (2012).

30. Yip, R. et al. Red cell membrane stiffness in iron deficiency. Blood 62, 99-106 (1983).

31. Yermiahu, T. et al. Quantitative determinations of microcytic-hypochromic red blood cell population and glycerol permeability in iron-deficiency anemia and beta thalassemia minor. Ann. Hematol. 78, 468-471 (1999).

32. Brandão, M. M. et al. Impaired red cell deformability in iron deficient subjects. Clin. Hemorheol. Microcirc. 43, 217-221 (2009).

33. Bunyaratvej, A., Butthep, P., Sae-Ung, N., Fucharoen, S. \& Yuthavong, Y. Reduced deformability of thalassemic erythrocytes and erythrocytes with abnormal hemoglobins and relation with susceptibility to Plasmodium falciparum invasion. Blood 79, 2460-2463 (1992).

34. Goma, J., Rénia, L., Miltgen, F. \& Mazier, D. Iron overload increases hepatic development of Plasmodium yoelii in mice. Parasitology 112(Pt 2): 165-168 (1996).

35. Koka, S. et al. Iron deficiency influences the course of malaria in Plasmodium berghei infected mice. Biochem. Biophys. Res. Commun. 357, 608-614 (2007).

36. Matsuzaki-Moriya, C. et al. A critical role for phagocytosis in resistance to malaria in iron-deficient mice. Eur. J. Immunol. 41, 1365-1375 (2011). 
37. Zhao, H. et al. Lipocalin 2 bolsters innate and adaptive immune responses to blood-stage malaria infection by reinforcing host iron metabolism. Cell Host Microbe 12, 705-716 (2012).

38. Bei, A. K. et al. A flow cytometry-based assay for measuring invasion of red blood cells by Plasmodium falciparum. Am. J. Hematol. 85, 234-237 (2010).

39. Theron, M., Hesketh, R. L., Subramanian, S. \& Rayner, J. C. An adaptable two-color flow cytometric assay to quantitate the invasion of erythrocytes by Plasmodium falciparum parasites. Cytometry A 77, 1067-1074 (2010).

40. lark, M. A. et al. RBC Barcoding Allows for the Study of Erythrocyte Population Dynamics and P. falciparum Merozoite Invasion. PloS One 9, e101041 (2014).

41. Simpson, J. A., Silamut, K., Chotivanich, K., Pukrittayakamee, S. \& White, N. J. Red cell selectivity in malaria: a study of multiple-infected erythrocytes. Trans. R. Soc. Trop. Med. Hyg. 93, 165-168 (1999).

42. Sriprawat, K. et al. Effective and cheap removal of leukocytes and platelets from Plasmodium vivax infected blood. Malar. J. 8, 115 (2009).

\section{Acknowledgements}

We gratefully acknowledge the individual donors who participated in the study. We also thank Michael Nicholson of Precision Biosciences for the use of the Beckman Quanta Flow cytometer and Steven R. Meshnick for critically reading the manuscript. The work was supported by the National Institute of Child Health and Human Development under award number U01HD061235 (to C.C.). The UNC Flow Cytometry Core Facility is supported in part by an NCI Center Core Grant number P30CA06086. S.M.T. is supported by the National Institute of Allergy and Infectious Diseases under award number K08AI100924.

\section{Author contributions}

M.A.C., M.M.G., C.C., N.F., M.A.E. performed experiments; R.S.K. and C.C. recruited patients; M.A.C., M.M.G., S.M.T., A.F., A.M.P., J.P. and C.C. analysed data; M.A.C., M.M.G., A.M.P., A.F., S.M.T. and C.C. wrote the manuscript.

\section{Additional information}

Supplementary Information accompanies this paper at http://www.nature.com/ naturecommunications

Competing financial interests: The authors declare no competing financial interests.

Reprints and permission information is available online at http://npg.nature.com/ reprintsandpermissions/

How to cite this article: Clark, M. A. et al. Host iron status and iron supplementation mediate susceptibility to erythrocytic stage Plasmodium falciparum. Nat. Commun. 5:4446 doi: 10.1038/ncomms5446 (2014). 